SPIE'S $12^{\text {th }}$ International Symposium on Smart Structures and Materials and $10^{\text {th }}$ International Symposium on NDE for Health Monitoring and Diagnostics, Sensors and Smart Structures Technologies for Civil,

Mechanical, and Aerospace Systems, San Diego, CA, 7-10 March, 2005, paper \# 5765-30

\title{
Efficient Electromechanical (E/M) Impedance Measuring Method for Active Sensor Structural Health Monitoring
}

\author{
Buli Xu \\ Mechanical Engineering Department, University of South Carolina \\ Columbia, SC 29208, xub@engr.sc.edu \\ Victor Giurgiutiu \\ Mechanical Engineering Department, University of South Carolina \\ Columbia, SC 29208, giurgiut@engr.sc.edu
}

\begin{abstract}
Electro-mechanical impedance method is emerging as an important and powerful technique for structural health monitoring. The E/M impedance method utilizes as its main apparatus an impedance analyzer that reads the in-situ $\mathrm{E} / \mathrm{M}$ impedance of the piezoelectric wafer active sensors (PWAS) attached to the monitored structure. Present-day impedance analyzer equipments (e.g. HP4194) are bulky, heavy and expensive laboratory equipment that cannot be carried into the field for on-site structural health monitoring. To address this issue, several investigators have explored means of miniaturizing the impedance analyzer making the impedance analyzer more compact and fieldportable.

In this paper we present an improved algorithm for efficient measurement of the E/M impedance using PWAS transducers. Instead of using a sine wave as the excitation signal to the PWAS and slowly changing its frequency, our method utilizes a chirp signal which is abundant in frequency components. By applying Fast Fourier Transform (FFT) to both the input and response signals, the impedance spectrum of the PWAS is acquired. The algorithm was implemented and tested in a real-time system, which consists of excitation signal generation module, voltage and current measurement module and digital signal acquisition module. The size and the implementation of the overall system using either a laptop or a digital signal processor (DSP) are also discussed. Finally, practical results are presented and comparatively examined.
\end{abstract}

Keyword: E/M impedance, SHM, Chirp signal, Fast Fourier Transform, PWAS, impedance analyzer

\section{INTRODUCTION}

Structural health monitoring (SHM) is a method of determining the health of a structure from the readings of an array of permanently-attached sensors that are embedded into the structure and monitored over time. SHM can be performed in basically two ways, passive and active. Passive SHM consists of monitoring a number of parameters (loading stress, environment action, performance indicators, acoustic emission from cracks, etc.) and inferring the state of structural health from a structural model. In contrast, active SHM performs proactive interrogation of the structure, detects damage, and determines the state of structural health from the evaluation of damage extent and intensity. Both approaches aim at performing a diagnosis of the structural safety and health, to be followed by a prognosis of the remaining life. The difference is, passive SHM uses passive sensors which only "listen" but do not interact with the structure. Therefore, it does not provide direct measurement of the damage presence and intensity. On the contrary, active SHM uses active sensors that can interact with the structure and thus determine the presence or absence of the damage. The methods used for active SHM resemble the methods of nondestructive evaluation (NDE), e.g., ultrasonics, eddy currents, etc., only that they use the embedded sensors. Hence, the active SHM could be seen as "embedded NDE". One widely used active SHM method employs piezoelectric wafer active sensors (PWAS), which send and receive Lamb waves and determine the presence of cracks, delaminations, disbonds, and corrosion. Due to its similarities to NDE ultrasonics, this approach is also known as "embedded ultrasonics". 
Electromechanical $(\mathrm{E} / \mathrm{M})$ impedance method is an embedded ultrasonic method which is emerging as an effective and powerful technique for SHM. E/M impedance method has several distinctive features ${ }^{1}$. Firstly, through PWAS permanently attached to the structure, it can directly measure the high-frequency local impedance spectrum of the structure. Secondly, because the high-frequency local impedance spectrum generated by E/M impedance method is much more sensitive to incipient damage than the low-frequency global impedance, this method is better suited for applications in structural health monitoring than other conventional methods. Lastly, the E/M impedance method utilizes as its main apparatus an impedance analyzer which reads the in-situ E/M impedance of the PWAS attached to the monitored structure.

In this paper we present an improved algorithm for efficient measurement of the $\mathrm{E} / \mathrm{M}$ impedance using PWAS transducers. The general aim is to go from the present-day bulky and expensive laboratory-size impedance analyzers to an integrated-circuit impedance analyzer that can be easily embedded into a structure for in-situ SHM. This objective is achieved by simplifying, miniaturizing, and integrating the functionality of existing laboratory-size electrical-impedance measuring equipment and by developing new implementation concepts utilizing state-of-theart methods and technologies in on-chip data acquisition, processing, and tele-transmission. We believe that this miniaturization is possible because:

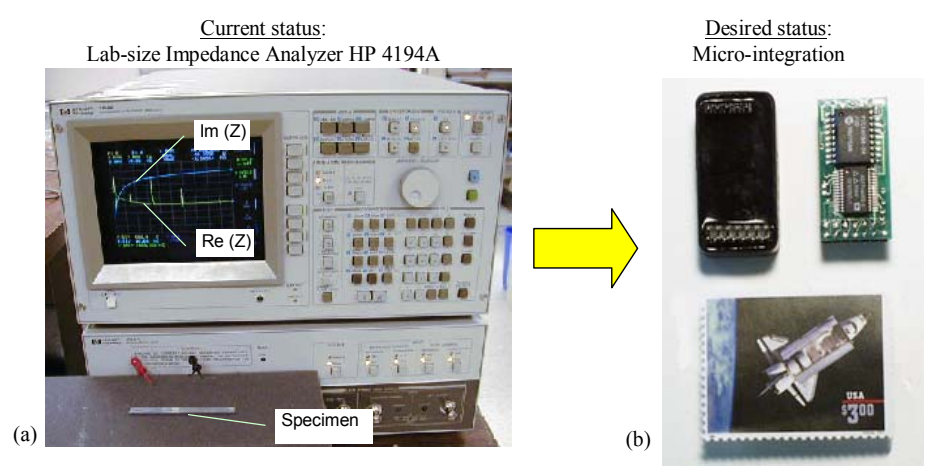

Figure 1 Miniaturization and integration of PWAS SHM equipment: (a) existing lab size impedance analyzer (bulky and relatively expensive); (b) miniaturized integrated electronics DAQ and tele-transmitter IC's (StrainLink, www.microstrain.com) that represent the miniaturization level targeted in this paper

1. The E/M impedance technique uses only a very small subset of all the functions and capabilities of laboratoryscale impedance analyzers such as the HP4194A instrument.

2. The design of existing laboratory-scale impedance analyzers is based on 1970's technology, while the design options considered in the present paper will utilize the latest developments in signal processing and microelectronics, many of them associated with the remarkable progress recently achieved in advanced digital design and wireless communication.

The implementation of this miniaturized impedance analyzer in active SHM systems will result in the high efficiency and seamless integration of active sensing, electronics, analysis, and diagnostics into a compact system in an unobtrusive way. In addition, by developing a lost-cost miniaturized impedance-measuring device, an on-line SHM system would be more compact and more easily used by the maintenance crews and SHM operators.

\section{STATE OF THE ART}

Several investigators have explored means of reducing the size of the impedance analyzer, to make it more compact, even field-portable. Alternative ways of measuring the E/M impedance, which are different from those used by the impedance analyzer, have also been researched.

Pardo de Vera and Guemes ${ }^{0}$ employed the electromechanical (E/M) impedance method to detect the damage in a GFRP composite specimen using a simplified impedance measuring technique. The simplified impedancemeasuring technique consisted in the use of an inexpensive laboratory-made RC-bridge instead of the costly HP4194A impedance analyzer (Figure 2a).

The disadvantages of using such a RC-bridge are: (a) additional instrumentation and processing need to be used to 
separate the signal into its real (in phase) part and imaginary (quadrature) part; (b) precise bridge balance needs to be initially attained in order to prevent the excitation signal from leaking into the output and masking the sensing signals; (c) the bridge needs to be manually balanced; (d) the frequency coverage is narrow with a single instrument.

Peairs, Park and Inman ${ }^{2,3,4}$ suggested a method of implementing impedance measurement utilizing an FFT analyzer and a small current-measuring circuit (Figure $2 \mathrm{~b}$ ). The approximated impedance $\left(Z_{\mathrm{PZT}}\right)$ is: $Z_{P Z T}=R_{s}\left(V_{i}-V_{0}\right) / V_{0}$.

The disadvantages of this method are: (a) inaccuracy shows up at high frequencies due to the performance limits of the amplifier; (b) a large laboratory-scale instrument-FFT analyzer is still needed; (c) currently available FFT analyzer is only appropriate for the analysis of signals in the range of a few millihertz to about a hundred kilohertz, which is insufficient for many SHM applications.

(a)

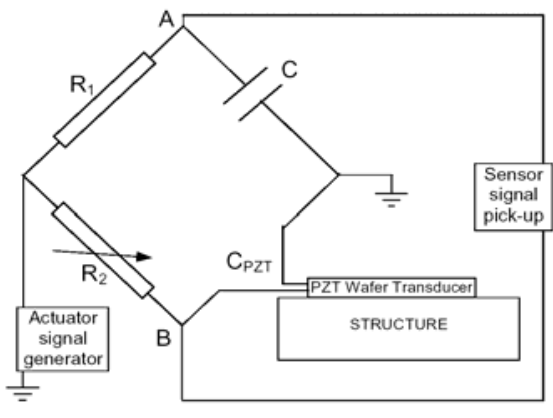

(b)

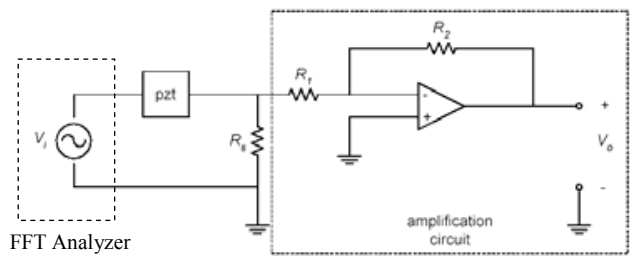

Figure 2 Previous results: (a) inexpensive RC bridge 0 for detecting the E/M impedance change of a PZT wafer transducer affixed to the health monitored structure; (b) Impedance approximating circuit with amplification $2,3,4$

\section{PREVIOUS WORK}

\subsection{The Concept}

In our previous work ${ }^{5,6}$, we presented a new concept of compact E/M impedance analyzer which utilizes standard low-cost multipurpose laboratory equipment: a function generator, a PCI DAQ card, a PCI GPIB card, a calibrated resistor $(100 \Omega)$ and a PC with LabVIEW software package (Figure 3).

(a)

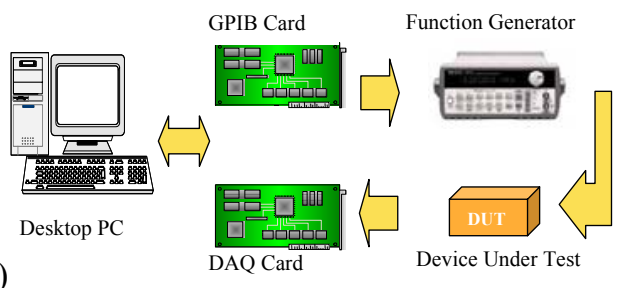

(b)

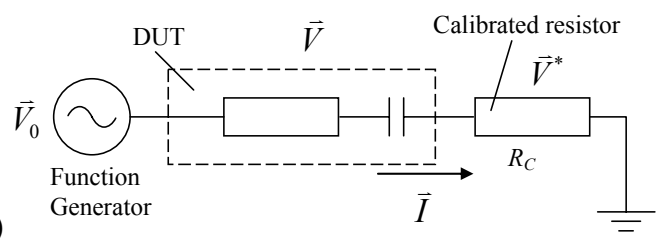

Figure 3 A proof of concept of miniaturized impedance analyzer: (a) hardware configuration; (b) measuring principle

The function generator outputs sinusoidal excitation $\vec{V}_{0}$ with its frequency sweeping in a predefined frequency range (from $f_{\text {start }}$ to $f_{\text {end }}$, say, $100 \mathrm{kHz}$ to $1 \mathrm{MHz}$ ). A two-channel DAQ card is employed to record the voltage $\vec{V}$ and $\vec{V}^{*}$ simultaneously. The current $\vec{I}$ flowing through the device under test (DUT) also flows through the already known low-value calibrated resistor $R_{c}$ (say, $R_{c}=100 \Omega$ ). Therefore, the current $\vec{I}$ can be calculated as $\vec{I}=\vec{V}^{*} / R_{c}$. Finally, the DUT impedance is given by

$$
Z=\frac{\vec{V}}{\vec{I}}=\frac{\vec{V}}{\vec{V}^{*}} R_{c}
$$

where $Z$ is the impedance of DUT, $\vec{I}$ is the current, $\vec{V}^{*}$ is the voltage across resistor $R_{c}$, and $\vec{V}$ is the voltage across the DUT. 


\subsection{Data Analysis method}

In Equation (1), vector $\vec{V}$ and $\vec{V}^{*}$ are unknown vectors, which can be represented in complex format when applying phasor method to calculate impedance $Z$. Three different data analysis methods, integration method, correlation method, and discrete Fourier transform method have been studied to measure the vectors in our previous work.

\subsection{Integration Approach}

Suppose a vector voltage $x(t)=A \sin \left(\omega t+\varphi_{0}\right)$ is multiplied by sine signal and cosine signal at the same frequency and then integrated over a time duration $T$, respectively. Where, $T$ is the period of $x$. The output will be the real part, $A \cos \left(\varphi_{0}\right)$, and the imaginary part, $A \sin \left(\varphi_{0}\right)$, of this harmonic voltage $x$ (Figure 4).

$$
\begin{aligned}
& A \cos \varphi_{0}=\frac{2}{T} \int_{0}^{T} A \sin \left(\omega t+\varphi_{0}\right) \sin (\omega t) d t \\
& A \sin \varphi_{0}=\frac{2}{T} \int_{0}^{T} A \sin \left(\omega t+\varphi_{0}\right) \cos (\omega t) d t
\end{aligned}
$$

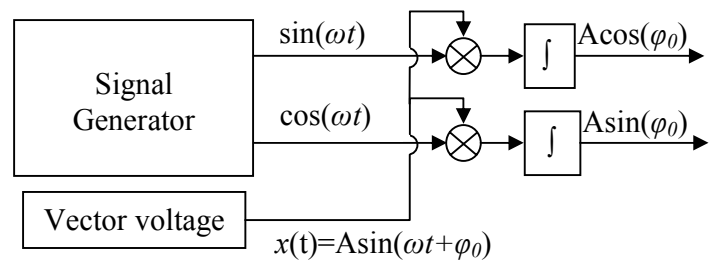

Figure 4 Schematic of the impedance measuring principle using the integration method

\subsection{CORRELATION APPROACH}

Consider two signals of the form:

$$
\begin{aligned}
& x(t)=A \sin \left(\omega t+\varphi_{0}\right)+N_{x}(t) \\
& y(t)=B \sin \left(\omega t+\varphi_{1}\right)+N_{y}(t)
\end{aligned}
$$

Where, $A$ is the amplitude of signal $x(t), B$ is the amplitude of signal $y(t), N_{x}(t)$ is the noise in signal $\mathrm{x}(t), N_{y}(t)$ is the noise in signal $y(t), \varphi_{0}$ is the initial phase of signal $x(t), \varphi_{1}$ is the initial phase of signal $y(t)$. Correlation of these two signals gives

$$
\begin{gathered}
\varphi=\arccos \frac{R_{X Y}(0)}{\sqrt{R_{X X}(0) \cdot R_{Y Y}(0)}} \\
A=\sqrt{2 R_{X X}(0)} \\
B=\sqrt{2 R_{Y Y}(0)}
\end{gathered}
$$

where $R_{X Y}$ is the cross-correlation of signals $x$ and $y, R_{X X}$ is auto-correlation of $x$. Equations (6), (7) and (8) permit the calculation of the amplitude ratio and relative phase difference of the two vectors in Equation (1).

\subsection{DISCRETE FOURIER TRANSFORM APPROACH}

Now consider a sinusoidal signal with certain initial phase $\varphi$, i.e.,

$$
x(t)=A \sin (2 \pi f t+\varphi)
$$

where $\varphi$ is the initial phase of the signal. Performing the DFT of $x(t)$ gives

$$
X(k)=A \frac{j}{2} e^{j \varphi}\left(e^{-j 2 \pi(q+k) n / N}-e^{-j 2 \pi(q-k) n / N}\right), k=0,1,2, \ldots, \mathrm{N}-1
$$

Note that: 


$$
\begin{cases}X(k)=\frac{A}{2} N \sin \varphi-j \frac{A}{2} N \cos \varphi, & k=q, \quad N-q \\ X(k)=0 \quad, \quad \text { others }\end{cases}
$$

Equation (11) allows us to calculate the amplitude, $A$, and phase, $\varphi$, of the signal $x$.

\subsection{Experimental results}

Comparison of the three methods (integration method, correlation method, and DFT method) to the laboratory-scale HP4194 impedance analyzer (which is the dedicated instrument for impedance measurement) has been performed. Among the three methods, integration method and DFT method show relatively better performance in the measurement of a free PWAS impedance spectrum. Here free means the PWAS is not mounted to any structure. Figure 5 and Figure 6 show the superposed free PWAS impedance spectrums obtained with the aforementioned three methods and the HP4194A laboratory impedance.

However, all the methods including HP4194A impedance analyzer use sinusoidal excitation signal at predetermined frequency values in the frequency range of interest. For measuring impedance at a given frequency, an excitation at this certain frequency is needed. That is to say, to plot an impedance spectrum of a PWAS with 401 frequency points, 401 different frequency excitations have to be generated, sampled and analyzed. This is not time efficient. New method is expected.

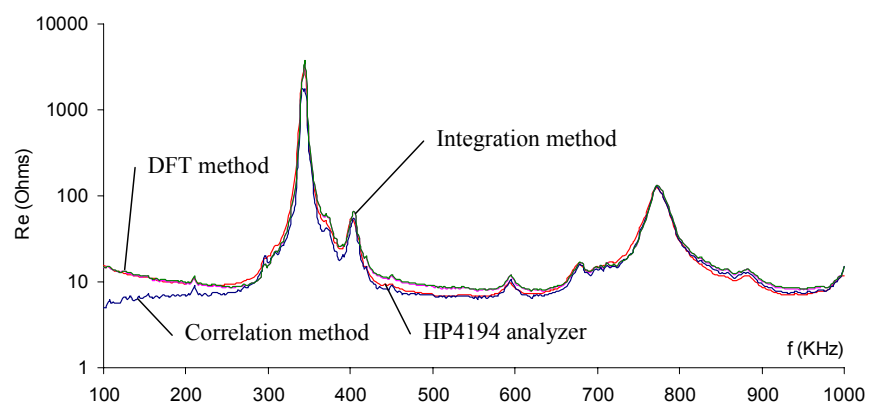

Figure 5 Comparison of measurement of real part of impedance of PWAS with different methods

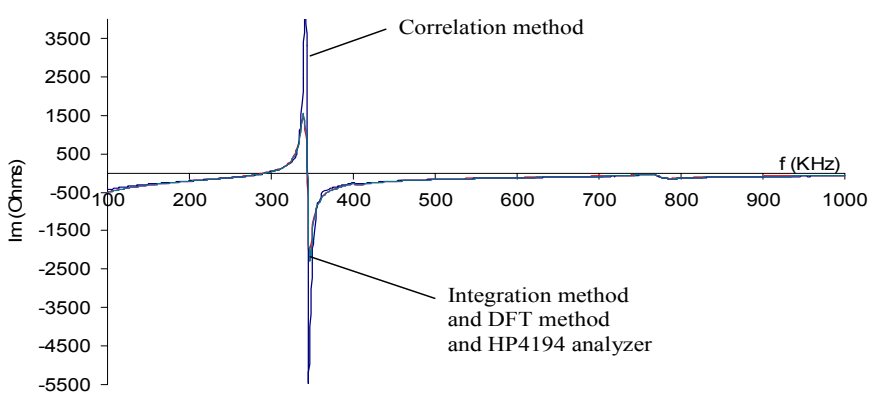

Figure 6 Comparison of measurement of imaginary part of impedance of PWAS with different methods

\section{PROPOSED IMPROVED APPROACH}

Therefore, an improved algorithm for impedance measurement is proposed. This improved algorithm is named FEMIA (Fast Electromechanical Impedance Algorithm) and made the object of an invention disclosure to the Univ. of South Carolina ${ }^{7}$. 


\subsection{The Concept}

For a linear system, by transforming the time domain excitation signal (voltage $[v(t)]$ ) and response signal (current $[i(\mathrm{t})])$ of the DUT to yield the frequency domain quantities $[V(j \omega)$ and $I(j \omega)]$, the admittance of DUT may be calculated as the transfer function of DUT ${ }^{8,9}$ (see Figure 7).

$$
Y(j \omega)=\frac{I(j \omega)}{V(j \omega)}
$$

Hence, the impedance of DUT is

$$
Z(j \omega)=\frac{V(j \omega)}{I(j \omega)}
$$

With this concept, the impedance spectrum of DUT can be acquired even within one excitation signal sweeping. The efficiency of the impedance measurement can be dramatically improved.

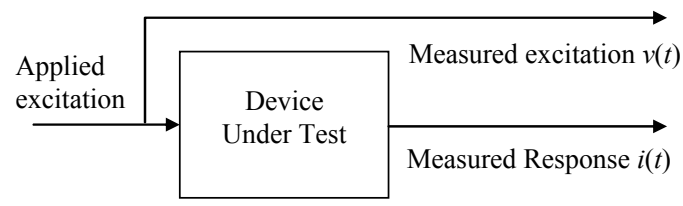

Figure 7 Configuration for impedance measurement using transfer function of DUT9

In addition to Equation (13), the impedance $Z(j \omega)$ can also be calculated from cross-power spectra of the input and the output ${ }^{9}$. Thus,

$$
Z(j \omega)=\frac{P_{v v}(j \omega)}{P_{v i}(j \omega)}
$$

Where, $P_{v i}(j \omega)$ is the cross-power spectrum of the excitation and the response signal, $P_{v v}(j \omega)$ is the auto-power spectrum of the excitation.

\subsection{Chirp signal}

From Equation (13), we can see that any arbitrary time domain excitation can be used to measure the system impedance provided that excitation is applied and the response signal is recorded over a sufficiently long time to complete the transforms over the desired frequency range.

Several investigators have tried various excitations to measure the electrochemical impedance. Sierra-Alcazar ${ }^{10}$, Doblhofer and Pilla ${ }^{11}$, and Creason and Smith ${ }^{12}$ used potential and current steps and various noise excitations to measure impedance. Searle. and Kirkup ${ }^{13}$ constructed an excitation waveform consisting of many frequency components to measure the impedance spectra of bioelectrodes. More recently, Darowicki ${ }^{14}$ studied the mathematical basis of using a continuous-frequency excitation and Gabor transformation to measure the impedance of the equivalent circuit of an electrode.

Here, we tried chirp signal as the excitation. The advantages of chirp signal are:

- Abundant in frequency components

- Frequency sweeping range can be easily controlled

- Sweeping speed can also be controlled by parameter $\beta$ (see Equation (18))

- Only one sweep of excitation is needed to acquire the impedance spectrum of device under test (DUT)

Consider the constant frequency sinusoidal signal

$$
x(t)=\operatorname{Re}\left\{A e^{j\left(\omega_{0} t+\phi\right)}\right\}=A \cos \left(\omega_{0} t+\varphi_{0}\right)
$$

We define the phase $\varphi(t)=\omega_{0} t+\varphi_{0}$ where $\varphi_{0}$ is initial phase, $\omega_{0}$ is phase velocity. The phase is a linear function of 
time. Furthermore, note that $d \varphi(t) / d t=\omega_{0}$. Then we define the general signal:

A linear chirp signal is produced when

$$
x(t)=\operatorname{Re}\left\{A e^{j \varphi(t)}\right\}
$$

$$
\varphi(t)=\pi \beta t^{2}+2 \pi f_{0} t+\varphi_{0}
$$

Computing the instantaneous frequency for the chirp, we have

$$
f_{i}(t)=\beta t+f_{0}
$$

Equation (18) is a linear function of time. The parameter $\beta=\left(f_{1}-f_{0}\right) / t_{1}$ is the rate of frequency change, which is used to ensure the desired frequency breakpoint $f_{1}$ at time $t_{1}$ is maintained. (Figure 8 )

(a)

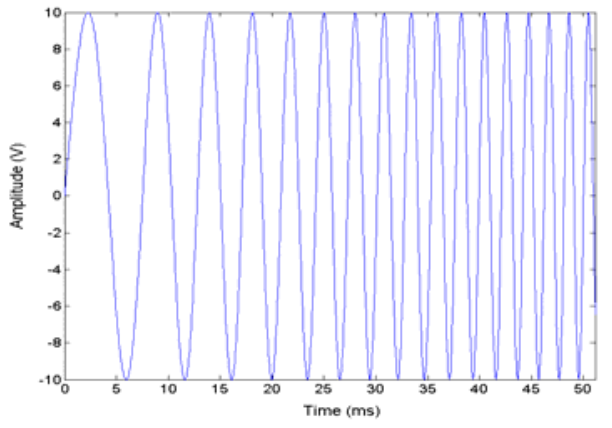

(b)

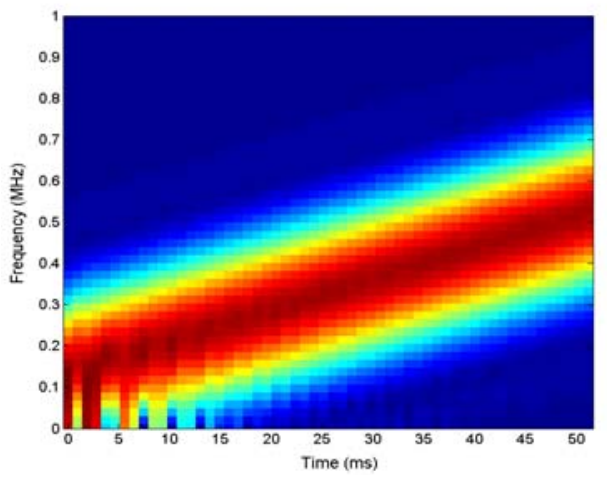

Figure 8 Chirp signal and STFT analysis of chirp signal: (a) chirp signal; (b) STFT of chirp signal

\subsection{Experimental results and discussion}

The testing of the improved algorithm for impedance measurement was performed in our proof-of-concept impedance analyzer (Figure 3). The function generator, which is controlled by a PC via GPIB card, outputs a linear chirp excitation with its frequency continuously sweeping within a defined frequency range $\left(f_{\text {start }}\right.$ to $f_{\text {end }}$, say, 100 $\mathrm{kHz}$ to $1 \mathrm{MHz})$.

The actual excitation and the response of the device under test (DUT) are recorded by a two-channel DAQ card synchronously. The impedance spectrum of the DUT equals Fast Fourier Transform (FFT) of the excitation over the FFT of the response signal (Figure 9).

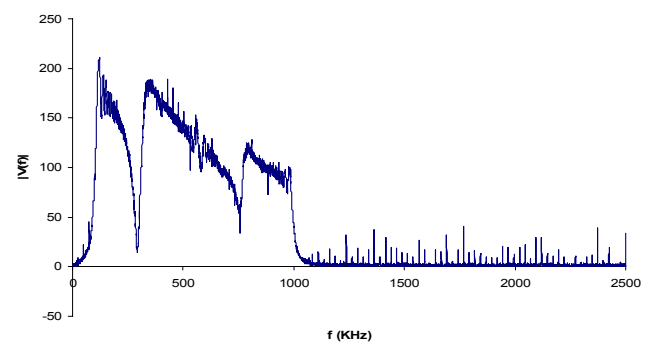

(a)

Figure 9 Magnitude of FFT of the measured excitation and FFT of the measured response signal with sampling frequency fs $=5 \mathrm{MHz}$ : (a) Magnitude of FFT of the measured excitation; (b) Magnitude of FFT of the measured response signal

Due to the internal resistance of function generator and capacitive character of the impedance PWAS, the FFT of measured chirp excitation differs from the FFT of chirp excitation in simulation.

The advantages of this proposed method are: 
- Only one sweep of excitation is needed

- Impedance spectrum measurement time depends on the range and rate of scanning of frequency

- The measurement is carried out for all frequencies points at the same time other than the "frequency by frequency" for previous methods

- The resulting impedance spectrum is continuous other than the discrete one measured by previous methods

Figure 10 shows the superposed impedance spectrums of a free PWAS obtained both by FEMIA and by HP4194A laboratory impedance analyzer.

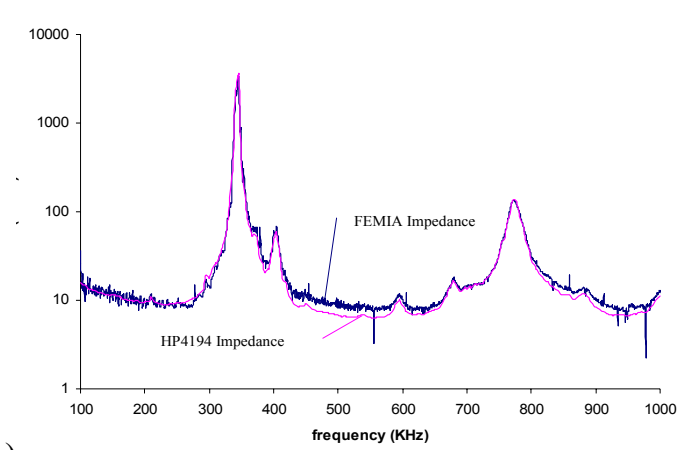

(a)

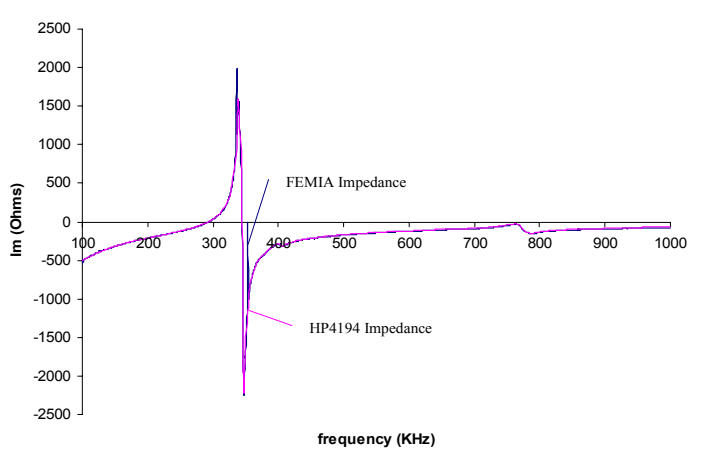

(b)

Figure 10 Comparison of measurements of PWAS impedance by HP4194 impedance analyzer and FEMIA: (a) Real part of PWAS impedance measurement; (b) Imaginary part of PWAS impedance measurement

The impedance spectrum acquired by the new algorithm is not as smooth as the one measured by HP4194 impedance analyzer. This is because:

- PWAS possesses large impedance at low frequency. This leads to low $\mathrm{S} / \mathrm{N}$ ratio of signal recorded by DAQ and error in the low-frequency part of impedance spectrum.

- The precision of this proposed method is mainly determined by the sampling frequency and the length of recorded excitation and response signal. In our experiment, due to the limitation of DAQ card buffer size, the sampling frequency used is only $5 \mathrm{MHz}$, and the sampling of DAQ only last for around $1 \mathrm{~ms}$.

- Both the recorded chirp excitation and the response signal are not continuous in time domain. After applying FFT to both of the two non-continuous time-domain signals, frequency leakage is introduced.

However, the preliminary results are still very encouraging. The algorithm improves dramatically the efficiency of impedance measurement. To improve precision of this algorithm, further research is still needed:

- Excitation signal construction. We only explored linear chirp signal as excitation signal in this paper. Some other forms of excitation signals, such as quadratic chirp (its frequency is a quadratic function of time), optimized/synthesized excitation signals for PWAS impedance measurement, deserve further research.

- The selection of an appropriate DAQ card with enough sampling frequency and buffer size is also important to improve the precision of the algorithm.

- Windowing. Apply windows to the recorded signals before doing FFT to reduce frequency leakage in this algorithm

In addition, to use this method, the device under test (DUT) is supposed to be a linear system. This method determines impedance as the ratio of the imposed input to the observed output without regard to the degree of causality between the two signals. For example, in a system exposed to noise, a component of the output signal power results from noise besides the applied input. For a nonlinear system, an excitation at frequency $\omega_{0}$ may results in harmonic distortion and a component of output power at the harmonic frequency points. To determine the validity of impedance measurement, a coherence function ${ }^{9}$ is always used. 


\section{CONCLUSION AND FURTHER WORK}

The proposed improved algorithm for $\mathrm{E} / \mathrm{M}$ impedance measurement has been explored in this paper. The new algorithm is much more efficient than the traditional methods. It can capture the whole impedance spectrum only within a sweep of excitation signal. Also, the resulting impedance spectrum is not discrete but continuous, which is different from those measured by traditional methods. However, this proposed method still has some limitations. The resulting impedance spectrum is not as smooth as the measurement from HP4194A impedance analyzer in our experiment. The error is due to the noise presenting in the measured system, hardware limitation and the property of the excitation signal. Further research on these parts is still needed.

In conclusion, the practical implementation of FEMIA consists of several blocks: (a) excitation signal generationfunction generator; (b) voltage and current measurements-DAQ card; and (c) digital signal acquisition and processing-PC and LabVIEW program. To further reduce the size and improve the mobility of the system, the PC can be replaced by a laptop. Accordingly, a much smaller PCMCIA DAQ card needs to be used. If function generator is controlled via RS232 cable, GPIB card can be eliminated from the system. We believe, by using a digital signal processor (DSP) for data analyzing, and a D/A converter for excitation signal generating, and an A/D converter for signals recoding, and a tele-transmitter module for data transmission, a small field-portable impedance analyzer for SHM could be developed.

\section{ACKNOWLEDGMENTS}

The financial support of National Science Foundation award \# CMS 0408578, Dr. Shih Chi Liu, program director, and Air Force Office of Scientific Research grant \# FA9550-04-0085, Capt. Clark Allred, PhD, program manager are gratefully acknowledged.

\section{REFERENCES}

1. Park, G.; Sohn, H.; Farrar, C. R.; Inman, D. J. (2003) "Overview of Piezoelectric Impedance-Based Health Monitoring and Path Forward", The Shock and Vibration Digest, Vol. 35, pp. 451-463, 2003

2. Pardo de Vera, C.; Guemes, J. A. (1997) "Embedded Self-Sensing Piezoelectric for Damage Detection," Proceedings of the International Workshop on Structural Health Monitoring, September 18-20, 1997, Stanford, CA $445-455$

3. Peairs, D.M.; Park, G; Inman, D.J. (2002) "Low Cost Impedance Monitoring Using Smart Materials", Proceeding of the First European Workshop on Structural Health Monitoring, Ecole Normale Superieure, Cachan (Paris), France, July 10-12, 2002

4. Inman, D.J. (2003) "Smart Materials in Damage Detection and Prognosis", Key Engineering Materials Vol. 245-246 (2003) pp3-16

5. Peairs, D.M.; Park, G; Inman, D.J. (2004) "Improving Accessibility of the impedance-Based Structural Health Monitoring Method”, Journal of Intelligent Materials Systems and Structures, Vol. 15, 129-139, Feb. 2004

6. Giurgiutiu, V.; Xu, B. (2003) "A self-processing integrated damage assessment sensor (SPIDAS) for structural health monitoring", USC-IPMO, Disclosure ID No. 00407 of 09/28/2003

7. Giurgiutiu, V.; Xu, B. (2004) "Development of a Field-Portable Small-Size Impedance Analyzer for Structural Health Monitoring using the Electromechanical Impedance Technique", SPIE's 11th Annual International Symposium on Smart Structures and Materials and 9th Annual International Symposium on NDE for Health Monitoring and Diagnostics, 14-18 March 2004, San Diego, CA. paper \# 5391-923

8. Giurgiutiu, V.; Xu, B. (2004) "FEMIA - Fast Electromechanical Impedance Algorithm", USC-IPMO, Disclosure ID No. pending of 04/28/2004

9. Macdonald, J. R.(1987), Impedance spectroscopy emphasizing solid materials and systems, Johm Wiley \& Sons, Inc., 1987

10. NI (1993), Application Note 041, “The Fundamentals of FFT-Based Signal Analysis and Measurement in 
LabVIEW and LabWindows", NI, Nov., 1993

11. Sierra-Alcazar, H.B.; Fleming, A.N., Harrison, J.A. (1978), "An Assessment of the measurements of impedance by analysis of a pulse using an on-line computer", J. Electroanal. Chem., 87, 399, 1978

12. Doblhofer, K.; Pilla, A.A. (1972), "Laplace Place Analysis of the Faradaic and Non-Faradaic Impedance of the Mercury Electrode", J. Electroanal. Chem., 39, 91, 1972.

13. Creason, S.C.; Smith, D.E. (1972), "Fourier Transform Faradaic admittance measurements: demonstration of the applicability of random and pseudo-random noise as applied potential", J. Electroanal. Chem., 36, 1, 1972

14. Searle, A.; Kirkup, L. (1999), "Real time impedance plots with arbitrary frequency components", Physiological Measurement, Vol. 20, 103-114, February 1999

15. Darowicki, K.; et al (2003), "Continuous-frequency Method of Measurement of Electrode Impedance", Instrumentation Science \& Technology, Vol 31, No. 1, pp 53-62, 2003 